\title{
Dinâmicas da agenda de políticas públicas na América Latina: oportunidades teóricas e empíricas
}

\author{
Frank R. Baumgartner 1 \\ Bryan D. Jones ${ }^{2}$ \\ Laura Chaqués Bonafont ${ }^{3}$ \\ 1 University of North Carolina at Chapel Hill, North Carolina - Estados Unidos da América \\ 2 University of Texas at Austin, Texas - Estados Unidos da América \\ ${ }^{3}$ Institut Barcelona d'Estudis Internacionals, Barcelona - Espanha
}

O Comparative Agendas Project (CAP) coleta, organiza e disponibiliza gratuitamente milhões de dados sobre os temas que têm chamado a atenção de governos em mais de 25 sistemas políticos de todo mundo. Os dados disponíveis compreendem um longo período desde a Segunda Guerra Mundial. Na expansão de seus projetos para a América Latina, os pesquisadores ligados ao CAP enfrentam, além de desafios comuns aqueles encontrados globalmente, outras adversidades particulares dos sistemas políticos adotados nas nações da região. Neste ensaio introdutório, exploramos os antecedentes do CAP e as oportunidades que se apresentam com sua expansão aos sistemas políticos latino-americanos.

Palavras-chave: comparative agendas Project; política pública; processo de políticas públicas; instituições políticas.

\section{Dinámica de la Agenda de Políticas Públicas en América Latina: oportunidades teóricas y empíricas}

El Comparative Agendas Project (CAP, por sus siglas en inglés) recopila, organiza y pone a disposición de forma gratuita millones de datos sobre los temas que han sido priorizados por parte de políticos y gobernantes en más de 25 sistemas políticos de todo el mundo. Estas bases de datos abarcan un período de tiempo largo, que en la mayoría de casos comienza tras el fin de la Segunda Guerra Mundial). Los investigadores que desarrollan proyectos siguiendo la metodología del CAP en América Latina tienen que hacer frente a los desafíos comunes que los investigadores han encontrado al analizar la agenda política, a otros exclusivos de los sistemas políticos de los países de esta región. En este ensayo introductorio, exploramos los antecedentes del CAP y las oportunidades que se presentan con su expansión a los sistemas políticos latinoamericanos.

Palabras clave: Comparative Agendas Project; agenda política; proceso de políticas públicas; instituciones políticas.

\section{Agenda dynamics in Latin America: theoretical and empirical opportunities}

The Comparative Agendas Project (CAP) collects, organizes, and makes freely available millions of bits of information concerning the objects of government attention over long periods of time (often back to the Second World War) for more than 25 political systems, worldwide. As researchers affiliated with the CAP expand their projects into Latin America, they confront some challenges similar to those from other regions, and some unique to their national political systems. In this introductory essay, we explore the background of the CAP and the opportunities posed by its expansion into Latin American political systems.

Keywords: comparative agendas project; agenda-setting; public policy; policy process; political institutions. 


\section{ANTECEDENTES DO COMPARATIVE AGENDAS PROJECT}

O Comparative Agendas Project (CAP) foi desenvolvido organicamente, a partir de um projeto de estudo sobre a formação da agenda governamental com foco nos EUA (US-based Policy Agendas Project). Na pesquisa realizada para seu livro Agendas and Instability in American Politics, publicado em 1993, Baumgartner e Jones constataram que era possível aprender muito avaliando pequenas informações durante longos períodos, de forma a rastrear as entradas e saídas de políticas públicas nas prioridades das agendas estudadas (Baumgartner \& Jones, 1993). Os autores estudaram temas como a energia nuclear, pesticidas e tabaco, rastreando a cobertura da mídia e as audiências conduzidas no Congresso dos EUA sobre esses tópicos, observando se a atenção era favorável ou crítica à indústria em questão. As técnicas quantitativas que começaram a ser usadas nessa iniciativa tornaram-se, em 1994, a base de um projeto de pesquisa que continua prosperando até hoje. O projeto com foco nos EUA atualmente conta com mais de um milhão de registros de atividades governamentais, como projetos de lei, votações nominais, estatutos promulgados, cobertura da mídia, ordens executivas presidenciais e decisões da Suprema Corte (Baumgartner \& Jones, 2015; Jones \& Baumgartner, 2005).

O CAP teve seu início quando Christoffer Green-Pedersen, da Universidade de Aarhus, visitou a Universidade de Washington em Seattle durante o ano acadêmico de 2004. Na ocasião, Green-Pedersen percebeu que poderia replicar o projeto norte-americano em seu país, a Dinamarca. No mesmo período, durante o ano acadêmico 2004-2005, Baumgartner foi pesquisador visitante na Europa, onde disseminou a abordagem do projeto americano a uma variedade de pesquisadores europeus. Stefaan Walgrave, da Universidade de Antuérpia, desenvolveu então um projeto semelhante na Bélgica e logo percebeu o valor de comparar seus dados a informações de outros países. A partir daí, o projeto se expandiu rapidamente abrangendo Reino Unido, Dinamarca, França, Bélgica, Espanha, Holanda, Canadá e Suíça, sendo que atualmente envolve projetos em mais de 25 sistemas políticos nos EUA, Europa e Ásia - mais detalhes sobre o desenvolvimento inicial da rede CAP pode ser encontrada no trabalho de Baumgartner, Breunig, e Grossman (2019a). A expansão do projeto para abranger os sistemas políticos da América Latina é, obviamente, o foco do presente artigo.

O ponto de conexão da rede do CAP se dá em torno de uma metodologia e de um conjunto de bancos de dados, não pela adoção de um único referencial teórico. A ideia do projeto é que os pesquisadores possam se desenvolver ao compartilhar uma infraestrutura. Assim como os astrônomos avançam em sua disciplina por meio da construção de telescópios maiores, é possível obter maiores insights sobre as ações dos governos em todo o mundo ao se expandir o escopo empírico dos estudos nessa área. As pesquisas no campo das políticas públicas geralmente se concentram em um problema de política de cada vez (ou em um número limitado deles). A ideia do CAP é reunir, exaustivamente, informações sobre todas as atividades de governo. Assim, em vez de estudar a política de saúde ou imigração, ou observar uma mudança específica nos regulamentos financeiros, política externa ou estratégias de defesa, pode-se aprender compilando informações sobre todas as ações de um governo, em todos os seus temas de interesse, durante um longo período. Os bancos de dados do projeto nos EUA (US Agendas Project) geralmente abrangem informações desde o período da Segunda Guerra Mundial. Alguns países têm bancos de dados que datam de períodos anteriores, embora a maioria não vá tão longe. Com uma longa visão histórica e um amplo escopo empírico, os estudiosos envolvidos no CAP buscam entender como os focos de atenção dos governos se movem de um tema a outro. A maioria dos pesquisadores está interessada em seus próprios países, mas a 
iniciativa facilita comparações entre sistemas, uma vez que há consistência nas categorias de temas usadas nos projetos relacionados ao CAP. Portanto, quando se acompanha a atenção às políticas de saúde na Espanha, é possível replicar facilmente essa análise e comparar a experiência espanhola com a francesa, a dinamarquesa, a britânica ou a suíça.

Ao tornar os dados equivalentes, o CAP permite fazer comparações e generalizações, mesmo sem impor uma perspectiva teórica específica. Talvez seja possível dizer que, ao fornecer bancos de dados quantitativos, o projeto promove uma abordagem quantitativa, e não qualitativa, ao estudo de políticas públicas. Mesmo assim, o projeto (como desenhado) tem sido frequentemente usado para fornecer o contexto quantitativo e o ponto de partida para estudiosos mais afeitos a abordagem qualitativa se aprofundarem em questões específicas, como por que ocorreu uma mudança em uma determinada política, em um particular momento histórico, em um país específico, a partir da iniciativa de um determinado grupo de lideranças, e com que argumentos e evidências essa mudança foi justificada. Afinal, os bancos de dados do projeto fornecem um sistema fácil de inclusão e indexação para todas as ações governamentais relevantes sobre qualquer tópico em particular. Portanto, embora a maioria das publicações relacionadas ao CAP tenha realmente adotado uma abordagem quantitativa, o projeto em si não se restringe a ela e pode ser usado como o primeiro passo de uma abordagem qualitativa e mais profunda - um exemplo de estudo com essas características é o conduzido por Engeli, GreenPedersen, e Larsen (2012), que se concentrou em comparações profundas da "política de moralidade" em vários países, a partir dos dados do CAP.

Talvez o CAP tenha tido mais influência na geração de novas áreas de estudo do que na forma como questões mais antigas têm sido estudadas. Por exemplo, Jones e Baumgartner (2005) buscaram compreender elementos da diversidade da agenda e de que maneira os orçamentos públicos se alteram. A diversidade da agenda refere-se ao grau em que os governos concentram oficialmente sua atenção coletiva (avaliado por meio de atividades legislativas, decisões judiciais, cobertura da mídia relacionada a ações governamentais ou outros indicadores) em apenas alguns tópicos considerados prioritários, ou se a atenção é difusa, cobrindo simultaneamente diferentes temas. É óbvio afirmar, mas difícil de medir, que os governos, por meio da divisão do trabalho, estejam simultaneamente envolvidos em muitas - dezenas de milhares - atividades diferentes. Quais instituições possuem a agenda mais diversa? Como os líderes políticos ou burocráticos lidam e gerenciam a complexidade esmagadora das diversas questões políticas que desafiam suas agências todos os dias? A coleta de dados permite fazer essa análise.

O campo da política orçamentária tem sido alterado pelos conjuntos de dados sobre orçamento oferecidos pelo CAP. Ao avaliar todos os orçamentos, por longos períodos e em todas as categorias de gastos, os estudiosos que adotam o CAP observaram poderosas semelhanças entre os processos orçamentários em todos os sistemas analisados até agora, levando à conclusão de que existe uma "lei empírica do orçamento público" que é consistente com as teorias da racionalidade limitada (Jones et al., 2009). A maneira pela qual os orçamentos se alteram muda muito pouco, na maioria das vezes e para a maioria das categorias. Entretanto, mudanças dramáticas podem ocorrer ocasionalmente (Jones, Baumgartner, \& True, 1998).

As bases de dados detalhadas associadas ao projeto têm permitido análises da dinâmica da política que até então não tinham sido exploradas minuciosamente. Por exemplo, Green-Pedersen e colaboradores examinaram o impacto das perguntas orais dos membros da oposição no parlamento dinamarquês, observando que, apesar de desprovidas de força constitucional ou influência direta 
sobre o governo, essas questões são eventualmente muito interessantes e geram notícias que, ao serem veiculadas, podem acabar pressionando o governo a agir (Green-Pedersen \& Mortensen, 2010; Seeburg, 2013). Chaqués Bonafont, Roqué, e Baumgartner (2015) mostraram dinâmica semelhante na Espanha.

Os estudiosos da opinião pública há muito tempo se interessam pelas dinâmicas da capacidade de resposta à essa opinião. Ainda, procuram compreender em que medida as mudanças políticas dos governos se relacionam com as mudanças na opinião pública e nos resultados das eleições (Erikson, Stimson, \& MacKuen, 2002; Stimson, 1999; Wlezien, 1995). Com o desenvolvimento das atividades do CAP, novas maneiras têm sido adotadas para abordar essas questões (Soroka \& Wlezien, 2010; Wlezien \& Soroka, 2012).

Sem precisar fazer uma revisão de todas as questões elucidadas pelos pesquisadores que fazem uso do CAP, o argumento exposto aqui é simples: um telescópio maior aumenta nossa capacidade de fazer descobertas. Muitos dos elementos apresentados acima foram recentemente explorados em um livro que descreve os dados relacionados ao CAP e os projetos associados a iniciativa (Baumgartner, Breunig, \& Grossman, 2019b).

\section{A ESTRUTURA DO BANCO DE DADOS DO CAP}

O livro de códigos inicial associado ao projeto de agenda governamental com foco nos EUA (US-based Policy Agendas Project) enumerou aproximadamente 235 tópicos de atenção em políticas públicas, formando um catálogo completo de atividades do governo dos EUA, com informações desde o período da Segunda Guerra Mundial. Quando Christoffer Green-Pedersen adotou o sistema pela primeira vez na Dinamarca, havia alguns ajustes a serem feitos. A Dinamarca é uma monarquia, e Baumgartner e Jones não haviam estabelecido nenhum código no sistema americano que estivesse apto a monitorar esse tipo de regime, ou um estado teocrático, por exemplo. Entretanto, todo país trata de questões de qualidade de seu sistema educacional, apoio à agricultura, gerenciamento costeiro, construção de barragens, infraestrutura de transporte, e assim por diante. Os ajustes de Geeen-Pedersen na lista de tópicos foram surpreendentemente poucos. No final, o livro de códigos dos tópicos principais do CAP difere do desenvolvido originalmente em base ao sistema político dos EUA, mas menos do que se poderia imaginar. Os governos de todos os lugares (pelo menos nos sistemas políticos explorados até agora) têm um conjunto comum de áreas de atividade. É digno de nota que alguns dos maiores ajustes foram na codificação detalhada do Pennsylvania Policy Database Project, que documentou uma série de atividades simplesmente não contabilizadas no governo federal dos EUA, entre elas o licenciamento de profissões ou a regulamentação das atividades de municípios, distritos e outras variadas entidades de governo local. Ou seja, os governos nacionais soberanos têm mais em comum entre si do que internamente, com suas próprias subunidades.

À medida que os vários projetos avançavam em diferentes países, às vezes adicionando novos códigos para resolver problemas específicos, uma situação crítica se tornou evidente. Observou-se que, quanto mais um sistema de codificação em um país necessita de modificações para lidar com suas peculiaridades, menor será o desenvolvimento de políticas públicas nesse local em comparação com os demais países. Consequentemente, sob a orientação de Sean Bevan, da Universidade de Edimburgo, a comunidade do CAP desenvolveu um livro de códigos baseado em 20 tópicos principais e 225 secundários. Os tópicos secundários são organizados hierarquicamente. Todos os sistemas dos países são construídos para que possam ser mapeados no sistema mestre do CAP, de forma que um seja diretamente comparável a todos os outros através das diretrizes do livro. Portanto, a maioria 
dos projetos nos países mantém dois sistemas de codificação: um sistema particular adaptado às necessidades específicas do país, e um sistema que codifica as observações sobre as políticas públicas de acordo com o livro de códigos inicial.

Um segundo requisito necessário para manter a comparabilidade é que, se novos tópicos e subtópicos forem adicionados, todos os temas antigos relevantes para o esse novo tópico deverão ser examinados ao longo de toda a série de observações e recodificados no novo sistema, se necessário. Esse requisito é denominado "comparabilidade retroativa", porque garante que, à medida que a série de atividades políticas avança, os tópicos permanecem comparáveis (Jones, 2016).

A American Political Science Association's Comparative Politics Section (seção de política comparada da American Political Science Association) observou na sua menção ao CAP na ocasião em que o projeto recebeu o prêmio 2019 Lijphart/Przeworski/Verba Dataset 2019 Award que o sistema "representa uma colaboração global que se baseia em um livro de códigos e abordagem harmonizados e comuns pelos quais os membros da equipe usam várias informações que permitem codificar prioridades, discussões, esforços e resultados de políticas públicas”. A citação continua observando: “The committee was impressed by the project's open and inclusive spirit, the latter evidenced by the project's extensive network of collaborators and various activities that bring together a diverse set of researchers using the CAP data. Owing to their Herculean efforts, their due diligence, and their commitment to data access and transparency, the project has left an indelible mark on the field of comparative politics".

\section{A CADA EXPANSÃO DO CAP, SURGEM NOVAS OPORTUNIDADES E PERSPECTIVAS}

A expansão do CAP em países da América Latina traz várias oportunidades. Além de permitir comparações mais diretas entre os processos políticos que ocorrem em países já incluídos no CAP e um novo conjunto de nações que até então não faziam parte dos bancos de dados, o contexto específico da política latino-americana também apresenta novas abordagens teóricas. Nesse caso, pode-se ter uma noção do futuro ao olhar o passado, uma vez que o histórico de expansões anteriores do CAP dá insights que orientam a expansão do projeto.

Um desses insights veio dos aprendizados com a primeira expansão do CAP - dos EUA para outros sistemas: os governos têm mais semelhanças do que diferenças. Quando o livro Agendas and Instability in American Politics (Baumgartner \& Jones, 1993) foi publicado, sua linguagem a respeito de "múltiplas localizações" e de complexidades do federalismo pode ter parecido a alguns estudiosos de abordagens comparativas como elementos particularmente americanos, com pouca aplicabilidade em sistemas políticos que não apresentam, por exemplo, separação de poderes, partidos políticos fracos ou fortes governos locais ou subnacionais. Entretanto, o livro tem sido amplamente utilizado como referência em outros contextos, dado seu foco na dinâmica de políticas e mudanças de quadro de longo prazo, o que se provou mais amplamente aplicável do que se imaginava.

Apesar de nascer nos EUA, o centro de gravidade geográfica do CAP está atualmente na União Europeia. A mudança para a Europa trouxe várias oportunidades e questões teoricamente interessantes. Dentre as principais está a atenção ao grau em que as ações da UE em Bruxelas afetam a formulação de políticas nacionais nos estados membros. A aplicação das diretrizes da UE implica tipicamente legislação a nível nacional, mas esses atos legislativos são mais ou menos obrigatórios. Esse processo levou a um menor envolvimento parlamentar em importantes negociações políticas, já que esse debate passa a ser feito no ambiente da UE entre os executivos representantes das nações, até que se apresente uma dessas diretrizes a partir de Bruxelas. Depois de lançada, há pouco que um partido 
político no legislativo nacional, seja de oposição ou membro da coalizão majoritária, possa fazer em relação a tal diretriz, além de aprovar a legislação necessária, sem emendas, para sua implementação. Estudar o impacto das regulamentações da UE tem sido uma parte importante da comunidade do CAP em todos os estados membros daquela região - o mesmo se observa no caso da Suíça, pois embora não seja membro da UE, o governo suíço ainda deve manter a conformidade com várias de suas regulamentações em favor da manutenção do mercado único. Essas questões estão longe das preocupações teóricas que deram início ao CAP, e são de fato irrelevantes para o projeto no contexto dos EUA, por exemplo. A natureza genérica e amplamente aplicável das bases de dados do CAP permite o estudo fácil e sistemático dessas questões, mesmo se elas não estavam previstas quando o projeto iniciou. Ou seja, ter um telescópio maior permite descobertas inusitadas, não previstas quando da construção do equipamento.

A natureza abrangente dos bancos de dados do CAP permite fazer perguntas inéditas na literatura, não imaginadas até então dadas as limitações das abordagens empíricas existentes. Jones e Baumgartner (2005) puderam então abordar questões como escassez de agenda, modelagem estocástica do orçamento e o conceito de "limiar de atenção" (que é influenciado não apenas pela gravidade de um problema subjacente, mas também pela gravidade cumulativa de outros problemas que estão, simultaneamente, na pauta de interesse do governo). Suas análises estão entre as primeiras a serem conduzidas com base nos bancos de dados do CAP, e levaram a literatura a áreas que não eram abordadas anteriormente.

Mais recentemente, Bryan Jones, Sean Theriault, e Michelle Whyman (2019) avançaram ainda mais, explorando as causas e consequências do chamado "Great Broadening", ou 'grande expansão' do escopo da política dos EUA no período pós-guerra. Os autores argumentam que a própria natureza do governo e do sistema político dos EUA foi dramática, radical e permanentemente alterada, em uma explosão de atividades em domínios políticos desconhecidos, no contexto do crescimento de atividades associadas à "Great Society" (grande sociedade) e em programas subsequentes, como a expansão dos regulamentos ambientais e a preocupação com direitos civis na década de 1960. Essas expansões alteraram as relações de poder e expandiram a abrangência das ações do governo a tal ponto que não havia como voltar atrás. Mais importante ainda, não teria sido possível avaliar essas mudanças no governo a partir de um único foco em indicadores como a carga tributária relativa ou o tamanho do orçamento federal como parcela do PIB. Em vez disso, os bancos de dados do CAP permitiram uma documentação de algo que não poderia ser visto de outra maneira: o surgimento do governo - ou seu espalhamento - em áreas onde anteriormente não era um ator importante. Depois que o governo chega nessas áreas não se observa mais recuo, e o debate político passou a lidar com a questão de como gerenciar o Estado administrativo.

\section{PROJETOS DO CAP NA AMÉRICA LATINA}

Atualmente, seis equipes de países diferentes - Argentina, Brasil, Chile, Colômbia, Equador e México - desenvolvem conjuntos de dados abrangentes para analisar a dinâmica da agenda em uma perspectiva comparativa (ver Quadro 1). Esses dados fornecem um instrumento único para a análise da agenda de políticas em três instâncias - parlamento, governo e mídia - em diferentes épocas e países. Esses conjuntos de dados cobrem um período de uma ou duas décadas, com algumas exceções (como o caso dos discursos do Presidente, no México. Esses registros abrangem até 50 anos, da década de 1960 até o presente). 
RAP | Dinâmicas da agenda de políticas públicas na América Latina: oportunidades teóricas e empiricas
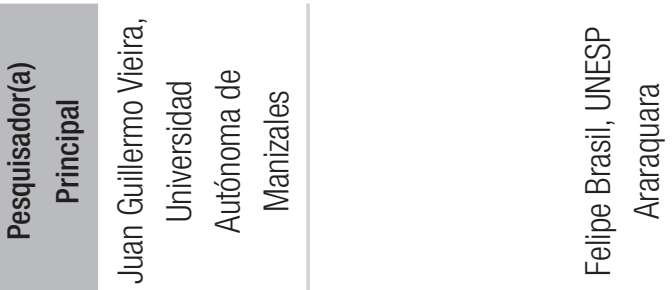

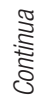
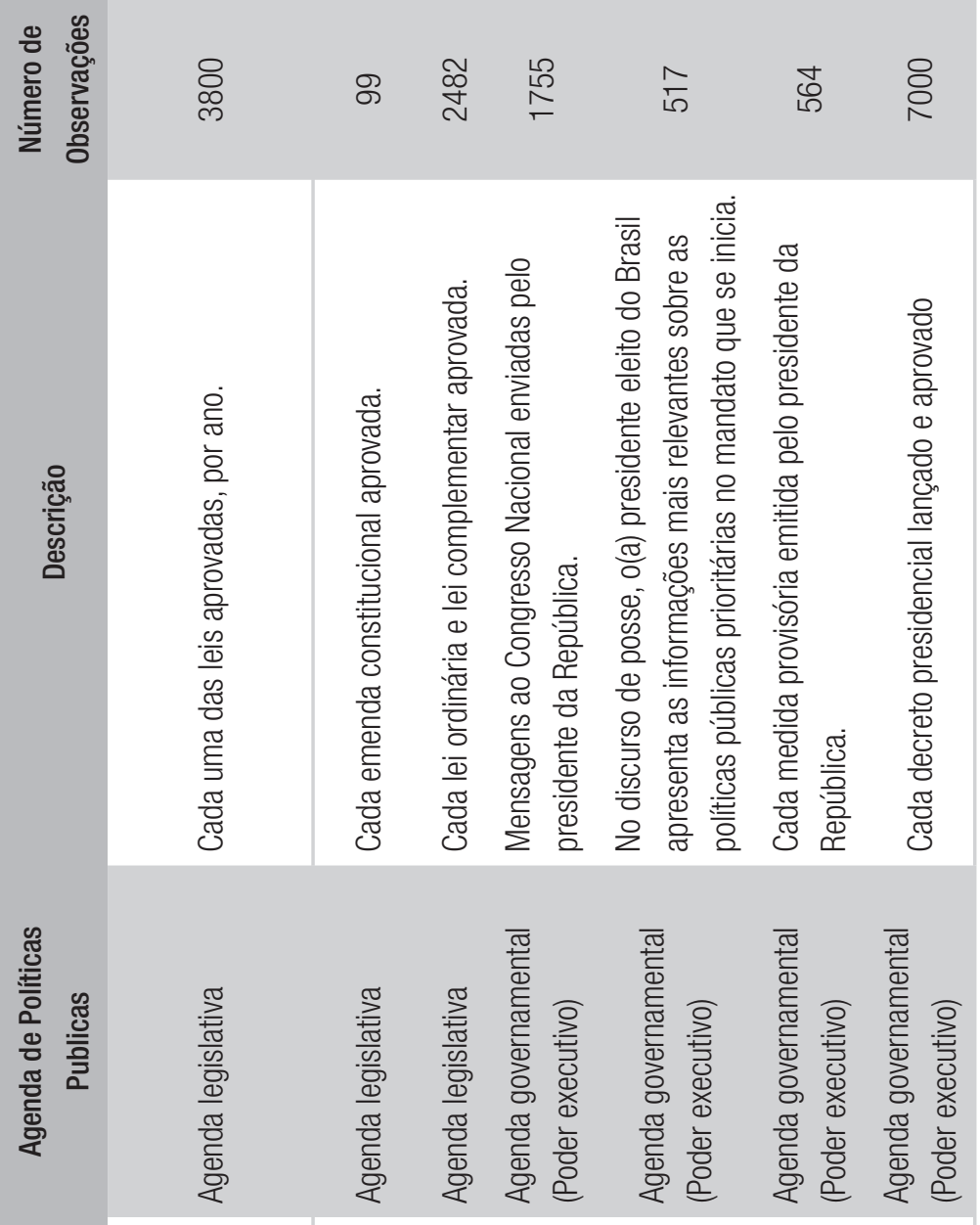

\begin{tabular}{|c|c|c|c|c|c|}
\hline 응 & 蒿 & $\stackrel{\sim}{\check{N}}$ & $\stackrel{\nabla}{\sigma}$ & $\frac{0}{2}$ & $\stackrel{\nabla}{\circ}$ \\
\hline
\end{tabular}

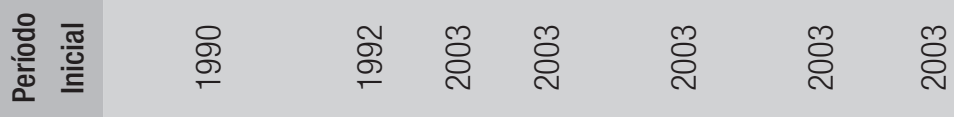


RAP | Dinâmicas da agenda de políticas públicas na América Latina: oportunidades teóricas e empíricas

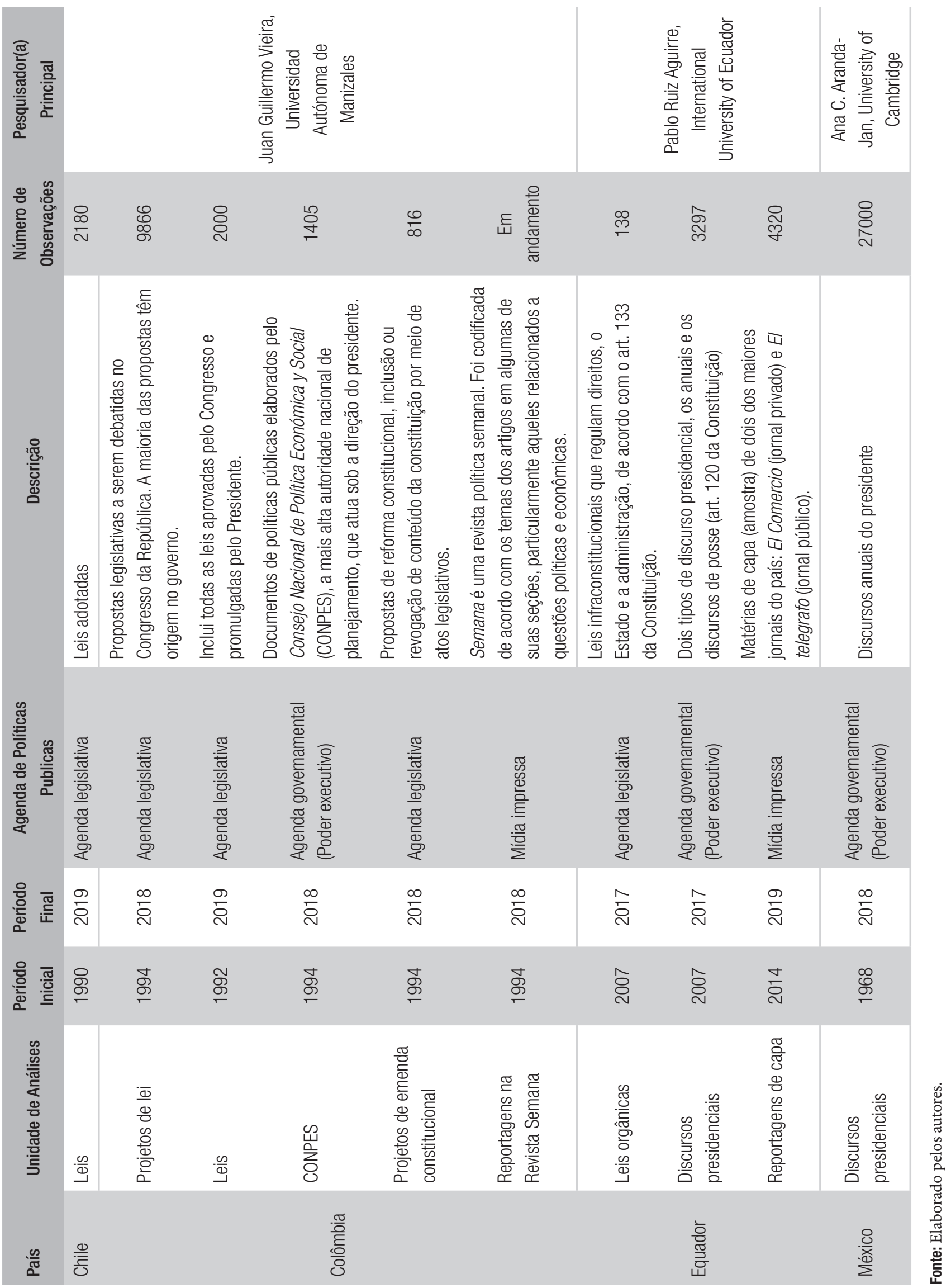


Para estudar as agendas do governo executivo, três equipes - no Brasil, Equador e México juntaram dados sobre o conteúdo político dos discursos presidenciais. Os discursos do poder executivo incluem, geralmente, propostas de políticas. No caso do Brasil, considera-se os discursos de posse, e as Mensagens ao Congresso Nacional como dados do executivo, já que não há um discurso anual do presidente como acontece em alguns outros países como no Equador e no México (Baumgartner et al., 2019a). Como em muitos outros países democráticos, no discurso de posse, o chefe do executivo apresenta o programa de governo do executivo, fornecendo informações importantes sobre suas prioridades políticas para a próxima legislatura. No caso dos discursos anuais, observa-se um padrão semelhante ao discurso "state of the nation" (estado da nação) dos EUA, que ocorre anualmente. Nesse caso, o Presidente informa a nação sobre as prioridades políticas para o resto da legislatura, bem como sobre a evolução e implementação do programa apresentado durante as eleições, informando sobre variações no conteúdo da agenda governamental desde o início do mandato.

Para a análise da agenda substantiva do executivo, na qual os chefes de governo não anunciam propostas, mas ações políticas efetivas, dois países, Brasil e Colômbia, desenvolveram conjuntos de dados diferentes. A equipe de pesquisadores do Brasil desenvolveu um conjunto de dados com informações sobre decretos presidenciais e medias provisórias, que são atos regulatórios adotados pelo poder executivo. São decisões formais tomadas em casos de necessidade extraordinária e urgente, quando circunstâncias excepcionais tornam impossível seguir o processo legislativo ordinário. A questão é se, como em muitos outros países, os presidentes usam cada vez mais esse instrumento legislativo para tomar decisões sobre questões que nada têm a ver com necessidades urgentes. No caso da Colômbia, a equipe do CAP desenvolveu um conjunto de dados sobre projetos de lei, que contém todas as informações sobre as propostas legislativas emitidas por parte tanto do executivo como de bancadas parlamentares. Como em muitos outros países, a supremacia do executivo no sistema político é ilustrada pelo alto percentual de projetos iniciados por esse poder, que finalmente são aprovados em leis, em comparação aos projetos iniciados pelo parlamento. Portanto, esse conjunto de dados fornece um instrumento relevante para estudar a relação assimétrica entre o executivo e o legislativo que caracteriza as democracias mais avançadas.

Para a análise das agendas parlamentares, as equipes dos países criaram diferentes conjuntos de dados, incluindo projetos de lei apresentados por bancadas parlamentares. Alguns países, como a Colômbia, também criaram um conjunto de dados sobre projetos de lei oriundos do parlamento. Como observado em outras democracias, esses projetos acabam sendo mais dispositivos para chamar a atenção do que atos projetados para gerar legislação. Além disso, diferentes equipes de pesquisadores do CAP criaram conjuntos de dados sobre leis orgânicas e/ou ordinárias na Argentina, Brasil, Chile, Colômbia e Equador. Por fim, os pesquisadores do Equador desenvolveram um conjunto de dados centralizado na agenda da mídia, coletando uma amostra de matérias nas primeiras páginas de dois dos jornais mais amplamente veiculados no país, El Comercio, que é um jornal privado, e El Telegrafo, um jornal público. Como em outros casos (ver Walgrave et al., 2019), a análise se concentra nas matérias de primeira página, que são importantes indicadores das questões que os meios de comunicação consideram prioritárias - um indicador quantificável da relevância das questões (e de sua capacidade de gerar notícias interessantes) do ponto de vista de editores e jornalistas.

Todos os bancos de dados foram codificados duas vezes por codificadores humanos, seguindo o livro de códigos do CAP. A primeira tarefa que as equipes de cada país realizaram antes de iniciar o procedimento de codificação, foi adaptar o livro de códigos às peculiaridades locais. Na maioria dos 
casos, isso significou adaptar as descrições existentes que melhor refletem as particularidades de cada país. Em alguns países, esse procedimento incluiu a criação de novas categorias destinadas a capturar aspectos que não têm equivalente em outros locais. A Colômbia é o único caso que realizou esse tipo de mudança, criando-se um novo código (50) para dar conta dos conflitos domésticos e dos processos de paz - um problema crucial na política colombiana nas últimas décadas.

Foram encontrados desafios na adoção da estrutura de agendas políticas comparadas (Comparative Policy Agendas framework) no contexto latino-americano, mas são tipos de desafio semelhantes aos observados nas experiências dos pesquisadores do CAP nos países europeus. A linguagem, a expansão para um novo conjunto de países, o contexto econômico, os atores internacionais, são questões parecidas com as já enfrentadas quando da migração do sistema dos EUA para a Europa. Ainda, é importante salientar questões como: será que existem códigos ou áreas de política que precisarão ser incluídos, ou algum que não será utilizado? O sistema de codificação específico do país pode ser facilmente integrado ao livro de códigos inicial do CAP? Será preciso pensar em bancos de dados ou indicadores que não são usados no contexto da UE ou dos EUA, como dívida, ajuda internacional ou indicadores comerciais? Deve-se incorporar as prioridades e os níveis de atividade desses atores da mesma forma que se faz com os atores da comunidade de credores internacional? Essas perguntas não são problemas teóricos ou simplesmente covariáveis contextuais que podem ser interessantes coletar. Isso seria como (pelo menos metodologicamente) incorporar diretrizes da UE na análise das dinâmicas das agendas dos governos dos estados membros.

É importante lembrar que o CAP é um sistema de medição. Ele captura variações nas agendas e nas ações políticas nos sistemas políticos de maneira sistemática, de modo que essas variações possam ser analisadas quantitativamente. O sistema pode ser usado a partir de diversas perspectivas teóricas e o trabalho qualitativo é essencial para complementar os estudos quantitativos de processos políticos baseados no CAP. Os pesquisadores do projeto mostraram que os governos tendem a adotar políticas semelhantes, mas em momentos e intensidades diferentes.

Essas variações não são autoexplicativas e sempre haverá espaço para análises qualitativas e estudos na área. A integração da forte tradição intelectual em estudos qualitativos observada na América Latina, com o trabalho quantitativo oriundo das equipes de pesquisa do CAP na região, apresenta um poderoso potencial acadêmico e prático.

\section{CONCLUSÕES}

A expansão do CAP para a América Latina apresenta inúmeras oportunidades teóricas e práticas. Teoricamente, expande para um novo contexto o leque de observações empíricas, permitindo testar com maior qualidade teorias novas e as já existentes. Assumindo-se que o passado funciona como preditor do futuro, serão abordadas um número maior de novas questões do que se pode atualmente imaginar. Uma área de investigação bastante óbvia é o impacto de reformas políticas neoliberais iniciadas ou incentivadas por certas agências de financiamento internacionais nas agendas e atividades domésticas dos países latino-americanos. Semelhante à avaliação do impacto das diretrizes da UE nos países membros que fazem parte do CAP, esses atores externos podem ter tido um impacto substancial na agenda doméstica e na distribuição de poder entre o executivo e o legislativo. No entanto, diferentes líderes latino-americanos adotaram as políticas de agências de financiamento internacionais com mais ou menos entusiasmo (ou, às vezes, recusaram-nas). Assim, a variedade 
RAP | Dinâmicas da agenda de políticas públicas na América Latina: oportunidades teóricas e empíricas

de respostas pode muito bem ser maior nos países da América Latina participantes do CAP do que observado anteriormente na UE.

Construir um telescópio maior é um convite a novas descobertas. O avanço do CAP por esse novo território tem o potencial de trazer muitos benefícios. 


\section{REFERÊNCIAS}

Baumgartner, F. R., Breunig, C., \& Grossman, E. (2019a). The Comparative Agendas Project: Intellectual Roots and Current Developments. In F. R. Baumgartner, C. Breunig, \& E. Grossman. (Eds.), Comparative Policy Agendas: Theory, Tools, Data. (pp. 3-16). New York, NY: Oxford University Press.

Baumgartner, F. R., Breunig, C., \& Grossman, E. (Eds.). (2019b). Comparative Policy Agendas: Theory, Tools, Data. New York, NY: Oxford University Press.

Baumgartner, F. R., \& Jones, B. D. (1993). Agendas and Instability in American Politics. Chicago, IL: University of Chicago Press.

Baumgartner, F. R., \& Jones, B. D. (2015). The Politics of Information. Chicago, IL: University of Chicago Press.

Chaqués Bonafont, L., Roqué, A. M. P., \& Baumgartner, F. R. (2015). Agenda Dynamics in Spain. London, UK: Palgrave Macmillan.

Engeli, I., Green-Pedersen, C., \& Larsen, L. T. (Eds.). (2012). Morality Politics in Western Europe: Parties, Agendas and Policy Choices. London, UK: Palgrave Macmillan.

Erikson, R. S., Stimson, J. A., \& MacKuen, M. (2002). The Macro Polity. New York, NY: Cambridge University Press.

Green-Pedersen, C., \& Mortensen, P. B. (2010). Who Sets the Agenda and Who Responds to it in the Danish Parliament? A New Model of Issue Competition and Agenda-Setting. European Journal of Political Research, 49(2), 257-281.
Jones, B. D. (2016). The Comparative Policy Agendas Projects as Measurement Systems. Journal of Public Policy, 36, 31-46.

Jones, B. D., \& Baumgartner, F. R. (2005). The Politics of Attention: How Government Prioritizes Problems. Chicago, IL: University of Chicago Press.

Jones, B. D., Baumgartner, F. R., Breunig, C., Wlezien, C., Soroka, S. N., Foucault, M. ... Walgrave, S. (2009). A General Empirical Law for Public Budgets: A Comparative Analysis. American Journal of Political Science, 53(4), 855-873.

Jones, B. D., Baumgartner, F. R., \& True, J. L. (1998). Policy Punctuations: US Budget Authority, 1947-95. Journal of Politics, 60(1), 1-33.

Jones, B. D., Theriault, S. M., \& Whyman, M. (2019). The Great Broadening: How the Vast Expansion of the Policymaking Agenda Transformed American Politics. Chicago, IL: University of Chicago Press.

Seeberg, H. B. (2013). The Opposition's Policy Influence through Issue Politicization. Journal of Public Policy, 33(1), 89-107.

Soroka, S. N., \& Wlezien, C. (2010). Degrees of Democracy: Politics, Public Opinion, and Policy. New York, NY: Cambridge University Press.

Stimson, J. A. (1999). Public Opinion in America: Moods, Cycles, and Swings (2nd ed.). Boulder, CO: Westview.

Wlezien, C. (1995). The Public as Thermostat: Dynamics of Preferences for Spending. American Journal of Political Science, 39(4), 981-1000.

Wlezien, C., \& Soroka, S. N. (2012). Political Institutions and the Opinion-Policy Link. West European Politics, 35, 1407-1432. 


\section{Frank R. Baumgartner}

https://orcid.org/0000-0003-4193-4152

É Richard J. Richardson Distinguished Professor de Ciência Política na University of North Carolina at Chapel Hill. E-mail: fbaum@email.unc.edu

\section{Bryan D. Jones}

É J. J. “Jake” Pickle Regent's Chair in Congressional Studies no Departamento de Governo na University of Texas at Austin.E-mail: bdjones@austin.utexas.edu

\section{Laura Chaqués Bonafont}

https://orcid.org/0000-0002-0724-4871

Professora de Ciência Política da University of Barcelona; Pesquisadora no Institut Barcelona d'Estudis Internacionals (IBEI). E-mail: lchaquesbonafont@gmail.com 